\title{
Effect of silybum marianum in treatment and prevention of hepatotoxicity in patients undergoing reinduction therapy for acute lymphoblastic leukemia (ALL)
}

\author{
Afshin Fathi ${ }^{1}$, Firouz Amani ${ }^{2,3 *}$ and Zhaleh Rouhzendeh ${ }^{3}$ \\ ${ }^{1}$ Department of Pediatrics, Ardabil University of Medical Science, Ardabil, Iran \\ ${ }^{2}$ Ardabil University of Medical Science, Ardabil, Iran, Postdoc in Department of Clinical Neuroscience, Karolinska Institutet, Stockholm, Sweden \\ ${ }^{3} \mathrm{GP}$, Ardabil University of Medical Science, Ardabil, Iran
}

\begin{abstract}
Using hepatotoxic drugs in induction phase for ALL patients increases liver enzymes and deal to some difficulties. So, treatment with herbal medicine such as Silymarin in monitoring serum levels of liver enzymes in ALL patients is effective. In a clinical trial study, we selected randomly 30 all patients and divided in two groups. One group treated with Silymarin and another group takes placebo for 56 days. Laboratory tests were done in patients on days 0 , 28 and 56 . The mean serum ALT and AST levels in two groups were similar in days 0 and 28, but in day 56 a significant decrease was seen in Silymarin group compared to placebo. Results showed that, the use of Silymarin in induction phase of ALL treatment significantly reduces liver enzymes and side effects of chemotherapy and be effective in prevention of hepatotoxicity in ALL patients.
\end{abstract}

\section{Introduction}

Acute Lymphoblastic Leukemia (ALL) is the most common cancer among children, one fourth of all cancers in children and $72 \%$ of all cancers in leukemia. The etiology of leukemia in children is unknown and probably multifactorial which genetic and environmental factors may involve in its occurrence. By recently research about treatment of childhood acute leukemia, the remission ratio of ALL patients has reached in 1950 from zero to $75-85 \%$. The main treatment of ALL is chemotherapy. Increasing medical knowledge about chemotherapy drugs combination, known effective dose and the use of short-term or long-term disability supportive treatment have reduced the number of these patients. In some patients undergoing chemotherapy, the occurred side effects lead to dose reduction and delay in treatment process and increase the risk of a relapse. The side effects can be included liver toxicity caused by L. asparaginase and doxorubicin in induction stage, that any protocol has not been presented on controlling liver enzymes and reducing liver toxicity in the induction stage of the the patients. Recently, the effect of Silymarin in liver enzyme controlling of these patients has been taken into consideration but there is no extensive information about the effect of treatment and prophylaxis of the drug. Silymarin is a herbal medicine and, also, has been approved in the protection of liver in liver disease such as acute and chronic hepatitis and liver cirrhosis. This is an anti-cancer medication and exacerbate the effects of vincristine. Silymarins leads to liver protection by protecting against free radicals, increasing antioxidants and endogenous enzymes, liver cells' membrane protection by reducing lipid peroxidation, stimulating protein synthesis, increasing the excretion of toxins and helping to liver regeneration [1-6]. The greatest impact of Silymarin is on reducing liver enzymes one month after starting medication, the use of Silymarin during the one month period of induction, in addition to the prevention of increase in liver enzymes in this period, reduces the hepatotoxic effects of drugs such as L. asparaginase, doxorubicin and 6-mercaptopurine [2-4] in the later stages of treatment and, consequently, facilitates the treatment. In Children Oncology, extensive studies on the effects of Silymarin in the prevention and treatment of hepatic and cardiac toxicity of chemotherapy drugs are being conducted [7]. The incidence rate of childhood cancer in Iran was 48 to 112 and 51 to 144 per million among girls and boys in multi geographical settings, respectively. The most common cancer in children from 0 to 14 years old were leukemia (incidence rate $=8$ to $62 /$ million), lymphoma, and central nervous system tumors. Tabriz is a big province in North West of Iran with population about 1,549,453 and more prevalent area for cancers [8].

According to Fathi et al., study, leukemia with 45 (54.2\%), CNS with $12 \%$ and Lymphomas with $8.4 \%$ were the most prevalent malignancies in Ardabil province and form leukemia cases $86.7 \%$ were acute lymphatic leukemia (ALL). The cumulative incidence rate was 95.4 patients per one million. More incidence rate was in year 2011 with 92.8 per 1000000 . The cumulative incidence rate in all children; girls and boys were 95.4, 85.9 and 10.3 per 1000000 of population. ALL with $47 \%$ were the prevalent cancer pathology in all age groups and also, most of ALL cases were in age groups 1-4 with 41\% [9].

The aim of this study was to investigate the impact of Silymarin

Correspondence to: Firouz Amani, Ardabil University of Medical Science,Ardabil,Iran, Postdoc in Department of Clinical Neuroscience, Karolinska Institutet, Stockholm, Sweden, Tel: +98 9144514670; Fax: +98 9144514670; E-mail: firouz.amani@ki.se

Key words: acute lymphoblastic leukemia, hepatotoxicity, silymarin

Received: May 20, 2016; Accepted: June 07, 2016; Published: June 10, 2016 
Fathi A (2016) Effect of silybum marianum in treatment and prevention of hepatotoxicity in patients undergoing reinduction therapy for acute lymphoblastic leukemia (ALL)

on the prevention of liver toxicity (hepatotoxicity) in ALL patients undergoing induction treatment in Tabriz Province north west of Iran.

\section{Methods and materials}

In this single blind clinical trial study, 30 patients with inclusion criteria were randomly selected from all the patients diagnosed with ALL that were hospitalized at cancer and blood sector of Ebne Sina hospital in Tabriz city and were under the treatment of induction based on vincristine and L. asparaginase. No known liver or diabetic diseases were observed in the patients. The samples were divided randomly in two Silymarin group and placebo group each with 15 patients. Silymarin drug with $5 \mathrm{mg} / \mathrm{kg} /$ day dose in the first day of chemotherapy and placebo with the same dose for 28 days were given to each group. For all patients CBC-diff, ALT AST, Bili (Total, Direct), FBS tests were checked on 0th, 28th and 56th days. $\mathrm{Na}$ and $\mathrm{K}$ levels in Serum were checked on 0th, 15th and 28th days. In the 7th and 28th days and after the beginning of induction, aspiration of bone marrow was performed and the rate of improvement was assessed. PT and PTT were measured on 4th and 22th days and blood pressure was measured on 0th and 28th days. Cardiac echo of these patients was performed on 0th and 28th days. Information included age, sex, type of acute lymphoblastic leukemia $(1 \mathrm{~L}, 2 \mathrm{~L}, 3 \mathrm{~L})$, bone marrow aspiration results, results of tests such as CBC-diff, ALT, AST, Bili (Total, Direct) FBS, echocardiogram, blood pressure measurement, side effects of chemotherapy and Silymarin were collected in a checklist and then analyzed. Drug side effects were fully explained to the patients before the study and consent form completed for all patients before admission to the study. This study was approval by the Ethics Committee of Ardabil Medical University (ARUMS1032) and registered in Iranian clinical trial (IRCT201408071325N2).

\section{Results}

$40 \%$ of the cases aged less than 3 years and $40 \%$ in the control group aged 3-6 years. The average age of the patients in the case group was $4.8 \pm 2.4$ and in the control group was $5.8 \pm 3.3$ years and the difference wasn't statistically significant. In both groups, 12 patients (80\%) have ALL Type L1 and 3 patients (20\%) have ALL Type L2. Of all patients, 21 patients (70\%) have chemotherapy side effects, of which 8 cases $(53.3 \%)$ were in the case group and 13 patients $(86.8 \%)$ in the control group and the difference between two groups was statistically significant ( $\mathrm{p}=0 / 046)$.The average serum levels of ALT, AST and FBS at $56^{\text {th }}$ day was significantly different between two groups, but there was no significant difference between two groups in the mean serum level of PLT in 56th day. There weren't significant differences between the two groups in the number of the ultrasonography performed at the beginning of the study and doing BMA in the $7^{\text {th }}$ day. Hepatomegaly frequency in both study groups was $66.7 \%$. Values of $\mathrm{Hb}$, total and direct bilirubin was similar between both groups in all the times. In case group, decreasing in the amount of FBS was significant at the beginning of the study compared to the $56^{\text {th }}$ day and in the control group, increasing in the amount of ALT at the beginning of the study compared to the $28^{\text {th }}$ and $56^{\text {th }}$ days were significant and other changes were not statistically significant (Figures 1-4).

\section{Discussion}

Of 30 patients with ALL and undergoing induction therapy, 15 patients treated with silymarin and 15 patients with placebo. In this study, only 2 patients (13.3\%) in the control group (13/33\%) having delay in the stages of chemotherapy and reducing in the treatment dose which was no significant difference between two groups. It has been

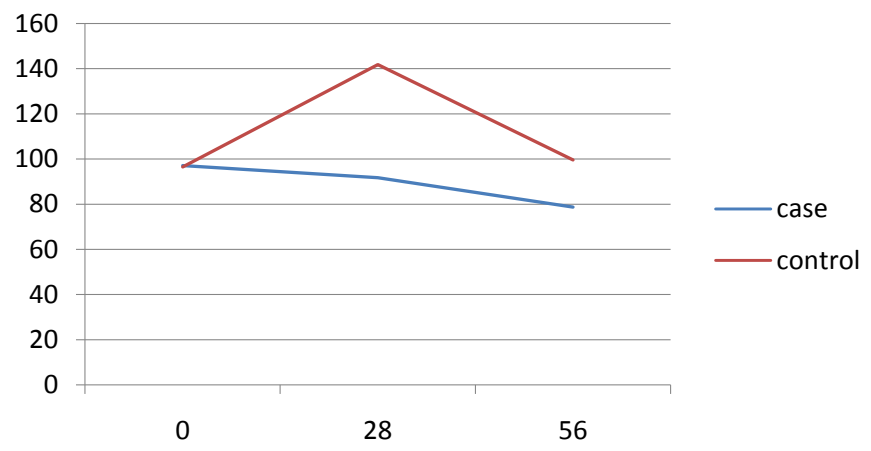

Figure 1. Trend of changes in FBS amounts in two groups by study time.

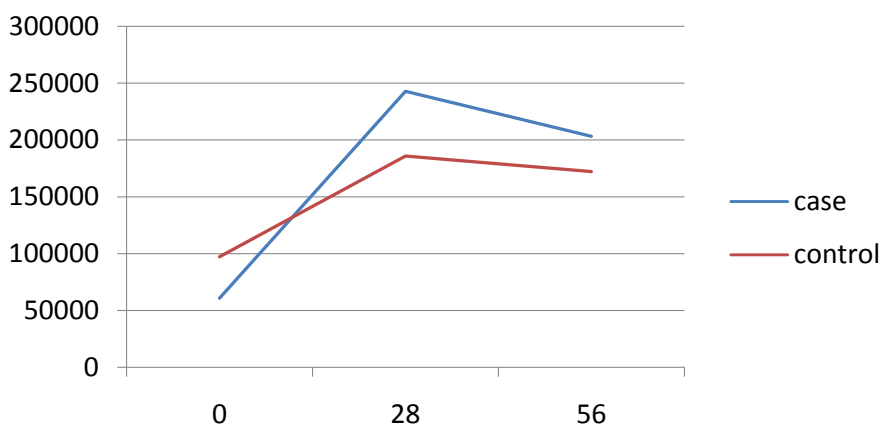

Figure 2. Trend of changes in PLT amounts in two groups by study time.

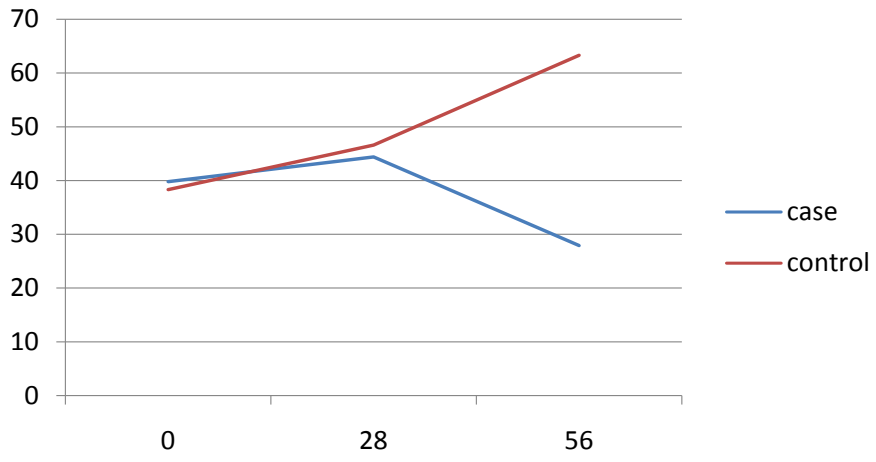

Figure 3. Trend of changes in AST amounts in two groups by study time.

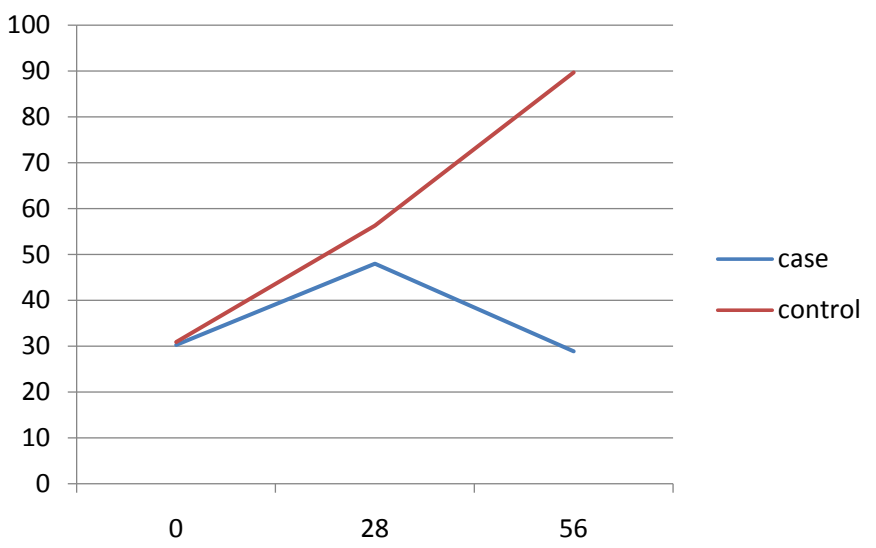

Figure 4. Trend of changes in ALT amounts in two groups by study time.

noted that in long-term and extensive studies, the difference might be significant because Silymarin, in addition to anti-inflammatory effects, 
Fathi A (2016) Effect of silybum marianum in treatment and prevention of hepatotoxicity in patients undergoing reinduction therapy for acute lymphoblastic leukemia (ALL)

has anti-carcinogenic and anti-metastasis effects too [10]. The mean difference of ALT and AST serum levels between two groups were not statistically significant on $0^{\text {th }}$ and $28^{\text {th }}$ days, but in 56th day there was significant. $(\mathrm{P}=0.005)$ Average serum levels of AST and ALT in the case group slightly increased at the end of induction compared to 0th day but after discontinuation of the drug during the next first month (56th day), the drug effects were observed and the mean serum levels became even lower than the first day that shows the fact that the greatest impact of the drug is in the during first month after treatment. Average serum level of ALT in the control group in three study times significantly have increased trend $(\mathrm{p}=0.007)$ but no significant increase in mean serum level of AST in this group which these results indicate a greater impact of Silymarin in reducing ALT compared to AST and Since ALT compared to AST is more specific liver enzyme, the above results could be expected. Average serum levels of Bili.Total, Bili.Direct, WBC, $\mathrm{Hb}$ and Plt between two groups was similar in all study times. Average serum levels of FBS between two groups on 0th and 28th days was similar, but on 56th day a significant difference was observed between two groups ( $\mathrm{p}=0.03$ ). Given that Silymarin is more effective on liver so no significant effect on $\mathrm{WBC}, \mathrm{Hb}$ and $\mathrm{Plt}$ is expected. 21 patients $(70 \%)$ were suffering from side effects of chemotherapy. Of these, 8 patients (53.3\%) were from the case group and 13 patients (86.7\%) from the control group and difference between two groups was significant $(p=0.046)$. Side effects included nausea and vomiting, neuropathy, diabetes and pancreatitis that all of them in the control group were higher and pancreatitis was not observed in the case group. It should be noted that about the side effects of Silymarin, particularly its longterm side effects, some studies have been conducted but further studies are recommended about it [11-14]. Hosseini et al. in a study showed that treatment with Silymarin in patients with type II diabetes have had significant effects on gylcemic parameters improvement of the patients which also in the present study the FBS values in Silymarin group were significantly decreased compared to the control group. McBride and et al in a study showed that Silymarin have been useful in the management and prevention hepatotoxicity in patients treated for AML which is similar to the results of this study [3]. Ebrahimpur et al., showed in a study that Silymarin improves the antioxidant indexes in type II diabetic patients and Silymarin had a significant effect on reducing liver enzymes which the results is similar to our study results [15].

\section{Conclusion}

Results showed that the use of Silymarin in the induction phase of ALL treatment can significantly reduce the liver enzymes and then the side effects of chemotherapy. Also using this herbal medicine can be effective in the prevention of hepatotoxicity in ALL patients and for more results, doing future studies about use of Silymarin in other stages of chemotherapy with more samples is suggested.

\section{Conflict of interest}

None-declared

\section{References}

1. Judith FM, Karen RR, David GP (2011) Acute lymphoblastic leukemia. In: Philip, A P., David G.P. Principles and Practice of Pediatric Oncology. Lippincott Williams \& Wilkins. Philadelphia 518-565.

2. Peter CA, Frank MB, Rochelle B, Susan MB (2011) General Principles of Chemotherapy. In: Philip, A.Pizzo, David G.Poplack. Principles and Practice of Pediatric Oncology. Lippincott Williams \& Wilkins. Philadelphia 279-355.

3. McBride A, Augustin KM, Nobbe J, Westervelt P (2012) Silybum marianum (milk thistle) in the management and prevention of hepatotoxicity in a patient undergoing reinduction therapy for acute myelogenous leukemia. J Oncol Pharm Pract 18: 360365 .

4. Ladas EJ, Kroll DJ, Oberlies NH, Cheng B, Ndao DH, et al. (2010) A randomized, controlled, double-blind, pilot study of milk thistle for the treatment of hepatotoxicity in childhood acute lymphoblastic leukemia (ALL). Cancer 116: 506-513.

5. Kalantari H, Shahshahan Z, Hejazi SM, Ghafghazi T, Sebghatolahi V (2011) Effects of silybum marianum on patients with chronic hepatitis C. J Res Med Sci 16: 287-290. [Crossref]

6. Huseini HF, Larijani B, Heshmat R, Fakhrzadeh H, Radjabipour B, et al. (2006) The efficacy of Silybum marianum (L.) Gaertn. (silymarin) in the treatment of type II diabetes: a randomized, double-blind, placebo-controlled, clinical trial. Phytother Res 20: $1036-1039$.

7. Biermer M, Berg T (2009) Rapid suppression of hepatitis C viremia induced by intravenous silibinin plus ribavirin. Gastroenterology 137: 390-391.

8. Mousavi SM, Pourfeizi A, Dastgiri S (2010) Childhood cancer in Iran. J Pediatr Hematol Oncol 32: 376-382. [Crossref]

9. Fathi A, Bahadoram M, Amani F (2015) Epidemiology of Childhood Cancer in Northwest Iran. Asian Pac J Cancer Prev 16: 5459-5462. [Crossref]

10. RaÅ kovi A, Stilinovi N, Kolarovi J, Vasovi V, Vukmirovi S, et al. (2011) The protective effects of silymarin against doxorubicin-induced cardiotoxicity and hepatotoxicity in rats. Molecules 16: 8601-8613. [Crossref]

11. El-Lakkany NM, Hammam OA, El-Maadawy WH, Badawy AA, Ain-Shoka AA, et al. (2012) Anti-inflammatory/anti-fibrotic effects of the hepatoprotective silymarin and the schistosomicide praziquantel against Schistosoma mansoni-induced liver fibrosis. Parasites \& Vectors 5: 9

12. Loguercio C, Festi D (2011) Silybin and the liver: from basic research to clinical practice. World J Gastroenterol 17: 2288-2301. [Crossref]

13. Jose MA, Abraham A, Narmadha MP (2011) Effect of silymarin in diabetes mellitus patients with liver diseases. J Pharmacol Pharmacother 2: 287-289.

14. Sagar SM (2007) Future directions for research on Silybum marianum for cancer patients. Integr Cancer Ther 6: 166-173. [Crossref]

15. Ebrahimpour Koujan S, Gargari BP, Mobasseri M, Valizadeh H, Asghari-Jafarabad M, et al. (2015) Effects of Silybum marianum (L.) Gaertn. (silymarin) extract supplementation on antioxidant status and hs-CRP in patients with type 2 diabetes mellitus: A randomized, triple-blind, placebo-controlled clinical trial. Phytomedicine 22: $290-296$.

Copyright: (C2016 Fathi A. This is an open-access article distributed under the terms of the Creative Commons Attribution License, which permits unrestricted use, distribution, and reproduction in any medium, provided the original author and source are credited. 